\title{
Separate phase-locking and coherent combining of two laser diodes in a Michelson cavity
}

\author{
G. Schimmel ${ }^{\text {a }}$, I. Doyen ${ }^{\text {a }}$, S. Janicot ${ }^{\text {a }, ~ L . ~ P . ~ R a m i r e z ~}{ }^{\text {a }}$, M. Hanna ${ }^{\text {a }}$, P. Georges ${ }^{\text {a }}$, G. Lucas-Leclin ${ }^{\text {a }}$, \\ V. Vilokkinen ${ }^{\text {b }}$, P. Melanen ${ }^{\text {b }}$, P. Uusimaa ${ }^{\text {b }}$, J. Decker ${ }^{\text {c }}$, P. Crump ${ }^{\text {c }}$, G. Erbert ${ }^{\text {c }}$, S. Bull ${ }^{\text {d }}$, S. Kaunga- \\ Nyirenda ${ }^{\mathrm{d}}$, E. C. Larkins ${ }^{\mathrm{d}}$ \\ ${ }^{a}$ Laboratoire Charles Fabry, Institut d'Optique, CNRS, Univ Paris-Sud, France; ${ }^{b}$ Modulight,
} Hermiankatu 22, FI-33720 Tampere, Finland; ${ }^{\mathrm{c}}$ Ferdinand-Braun-Institut, Leibniz-Institut für Höchstfrequenztechnik, 12489 Berlin, Germany; ${ }^{\mathrm{d}}$ School of Electrical and Electronic Engineering, University of Nottingham, University Park, Nottingham, United Kingdom

*gaelle.lucas-leclin@institutoptique.fr

\begin{abstract}
We describe a new coherent beam combining architecture based on passive phase-locking of two laser diodes in a Michelson external cavity on their rear facet, and their coherent combination on the front facet. As a proof-of-principle, two ridge lasers have been coherently combined with $>90 \%$ efficiency. The phase-locking range, and the resistance of the external cavity to perturbations have been thoroughly investigated. The combined power has been stabilized over more than 15 min with an optical feedback as well as with an automatic adjustment of the driving currents. Furthermore, two high-brightness high-power tapered laser diodes have been coherently combined in a similar arrangement; the combining efficiency is $70 \%$ and results in an output power of $4 \mathrm{~W}$. We believe that this new configuration combines the simplicity of passive self-organizing architectures with the optical efficiency of master-oscillator power-amplifier ones.
\end{abstract}

Keywords: coherent combining, passive phase-locking, diode lasers, external cavity.

\section{INTRODUCTION}

Coherent beam combining (CBC) of diode lasers consists in the superposition of individual laser sources, by constructive interference between them. This makes it possible to produce a single high-power laser beam with excellent spectral and spatial properties [1]. For CBC to be effective, the individual laser elements must have a proper phase relationship that remains constant over time, which can only be achieved in an arrangement that forces the required phase relation between the emitters. Additionally, only laser beams with identical transverse profiles can be coherently combined thus, CBC architectures are based on single-transverse mode lasers. Over the years, different approaches have been investigated: either active phase-locking of amplifiers seeded by a single-frequency laser split into $\mathrm{N}$ beams and amplified in parallel in the individual amplifiers, or passive self-organization of emitters in a common laser cavity [2]. The feasibility of such architectures has been brilliantly demonstrated with fiber and solid state lasers, showing impressive performance in the past few years.

With diode lasers and amplifiers, the highest output power and the largest number of phase-locked semiconductor amplifiers (respectively $40 \mathrm{~W}$ and 218) have been demonstrated in the Master-Oscillator Power-Amplifier (MOPA) configuration by Creedon, Redmond and their co-workers at MIT Lincoln Laboratory [3][4]. They have used arrays of individually-addressed slab-coupled waveguide amplifiers (SCOWA), which typically provide an output power of $1 \mathrm{~W}$ per device in a diffraction-limited profile. The MOPA architecture leads intrinsically to a high electrical-to-optical (E-O) efficiency, but requires non-standard devices and real-time active correction of the phase. On the other hand, different external cavity setups have been described in the literature, based on self-imaging [5] and interferometric architectures to force the passive phase-locking of lasers. Nevertheless, with such passive cavities the coherence of the laser beam drops as the operating current increases and the need for a high enough optical feedback on the front facet of the lasers reduces the E-O efficiency of such phase-locking configurations. Active correction of the lasers has been demonstrated to allow phase-locked operation at high currents, but still with a limited E-O efficiency [6].

In the following we investigate a new $\mathrm{CBC}$ architecture using a common external cavity on the back side of the lasers for phase locking, while the coherent beam superposition of the phase-locked beams is realized on the front side. This 
technique leads to a separation of the phase-locking stage - which takes place in the common external cavity - and the beam combining stage - which is achieved outside the cavity. As a consequence, the E-O efficiency of the phase-locked laser array is increased as compared to standard external cavity configurations. As a proof-of-principle, this configuration is demonstrated with two lasers in a Michelson-type external cavity. Detailed analysis of the externalcavity operation is performed with truly single transverse ridge waveguide lasers. With the aim to demonstrate the capability of such a setup for high power operation, it is also applied to two high-brightness high-power tapered devices.

\section{DESCRIPTION OF THE LASERS}

Two different kinds of lasers have been used for our phase-locking experiments: firstly, low-power ridge devices which ensure a purely single-transverse transverse profile; secondly, high-brightness tapered devices, in which a ridge section is used as a spatial filter to force nearly diffraction-limited operation [7].

\subsection{Ridge lasers}

The ridge waveguide lasers, manufactured by Modulight, are $2 \mathrm{~mm}$ long with a $4 \mu \mathrm{m}$ wide ridge which ensures a single transverse mode operation. The lasers have been specifically designed for operation with an external cavity. . The design is based upon a super large optical cavity with low fast-axis divergence to permit efficient coupling to and from the external cavity. Such cavities support many higher order vertical modes, however, which may be excited by backcoupled light (e.g. due to optical aberations, pointing error, etc.). The excitation of these parasitic vertical modes is suppressed by using an optical trap to reduce their modal gain relative to that of the fundamental mode. The RW lasers here employ a $2.4 \mu \mathrm{m}$ wide InGaP waveguide with an InGaAsP OT layer in the n-waveguide, whose position was optimized to give the best trade-off between the near-field width and higher order mode suppression [8]. With a $\mathrm{R}=95 \%$ reflection on the back facet and a standard anti-reflection $(\mathrm{R}=3 \%)$ coating on the front one, the output power of these lasers reaches $0.76 \mathrm{~W}$ at $1 \mathrm{~A}$. The maximum $45 \%$ electric-optic conversion efficiency is achieved at $0.4 \mathrm{~A}$ for an output power of $0.34 \mathrm{~W}$. The transverse beam profile is single-mode in both directions, with vertical and horizontal far-field angles of $29^{\circ}$ and $7^{\circ}(\mathrm{FWHM})$, respectively.

In the following experiments, devices with an anti-reflection coating on the back facet $(\mathrm{R} \leq 0.1 \%)$ instead of the HR coating have been used. Each laser has been first evaluated independently using an external cavity on the back facet consisting of a collimating lens and a high-reflectivity mirror. The laser threshold was $\sim 50 \mathrm{~mA}$, and the output power reached $0.2 \mathrm{~W}$ at $250 \mathrm{~mA}$, with a slope efficiency of $0.95 \mathrm{~W} / \mathrm{A}$. These results are close to those obtained with $\mathrm{HR} / \mathrm{AR}$ lasers. Nevertheless, thermal management of devices with access to both facets is less efficient than for standard lasers, which limits the maximum extracted power.

Finally, we were interested in the phase shift applied to the laser beam during single-pass propagation through the devices and its evolution with the driving current as a consequence of the local heating of the laser. It has been measured in a Mach-Zehnder interferometer, with a AR/AR device used as an amplifier in one arm (Figure 1). The reference source was a single-frequency laser source tuned at $950 \mathrm{~nm}$. When the amplified and the reference beams are superimposed with a slight angle, we observe a high-contrast fringe pattern which shifts with the driving current of the amplifier. This transverse evolution gives a measurement of the phase-to-current response of the device under test. Here, the phase shift is typically $\pi / 38$ per $\mathrm{mA}$ for single-pass transmission through the 2 -mm long device (Figure 2).

\subsection{Tapered lasers}

We have also characterized tapered laser devices specifically developed by the Ferdinand-Braun-Institut, Leibniz-Institut für Höchstfrequenztechnik. Details of the device design and assembly are given in [7]. These lasers contain a $2 \mathrm{~mm}$ long ridge section, and a $4 \mathrm{~mm}$ long tapered section ( $6^{\circ}$ taper angle) and are mounted here p-side up on C-mount. The Cmount dimension is matched to the total chip length of $6 \mathrm{~mm}$, to allow optical access to both facets. Electrical contact to the tapered region is made via a custom-dimensioned $\mathrm{CuW}$ submount, which is soldered to the $\mathrm{p}$-side of the device for uniform pumping and improved cooling. The ridge is contacted separately via wire-bonds. The two sections are separately driven by currents $I_{R}$ and $I_{T}$, respectively When prepared with $R=96 \%$ coating on the rear facet and $1 \%$ on the front facet, the lasers emit $4 \mathrm{~W}$ for a ridge current $\mathrm{I}_{\mathrm{R}}=400 \mathrm{~mA}$ and a taper current $\mathrm{I}_{\mathrm{T}}=6.5-7 \mathrm{~A}$. The vertical far-field is typically $14^{\circ}$ (FWHM), with the lateral beam profile on the tapered facet is $15^{\circ}$ wide at $4 \mathrm{~W}$ output power (FW95\%). $80-85 \%$ of the total power is within the central diffraction-limited lobe at $4 \mathrm{~W}$, leading to a beam quality of $\mathrm{M}^{2}=1.4$ at the $1 / \mathrm{e}^{2}$ level. 
Similarly to the characterizations done with the ridge lasers $(\$ 2.1)$, devices with $0 \%$ (rear facet) and $0.5 \%$ (front facet) anti-reflection coatings were used in an external cavity configuration. There was no significant difference in electro-optic performance between the results achieved from the devices in external cavity and from the HR/AR lasers.

Finally, a true amplifier, with $\mathrm{R}=0 \%$ antireflection coatings on both facets but the same geometry, was used in the Mach-Zehnder interferometer (Figure 1). The phase-shift induced by propagation through the amplifier was measured as a function of the operating currents in the ridge and tapered sections for two different injected powers $P_{\text {in. }}$ As for the ridge amplifiers, the phase-to-current response of the tested device exhibits a slight non-linear behavior at low current; the single-pass phase shift is typically equal to $\pi / 35$ per $\mathrm{mA}$ (Figure 3 ).

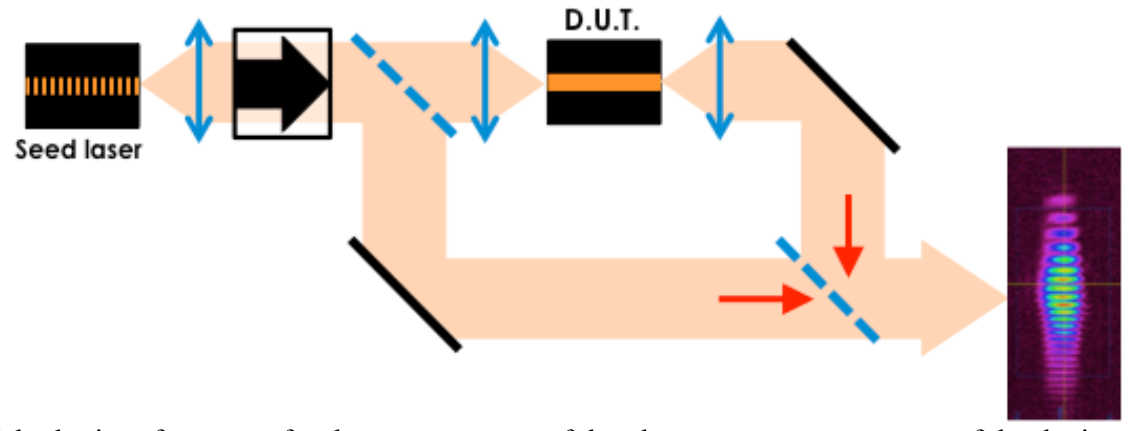

Figure 1: Mach-Zehnder interferometer for the measurement of the phase-to-current response of the device under test (D.U.T.) and typical interference fringe pattern produced by the amplified and free-space beams along the fast-axis direction, using a ridge waveguide amplifier.

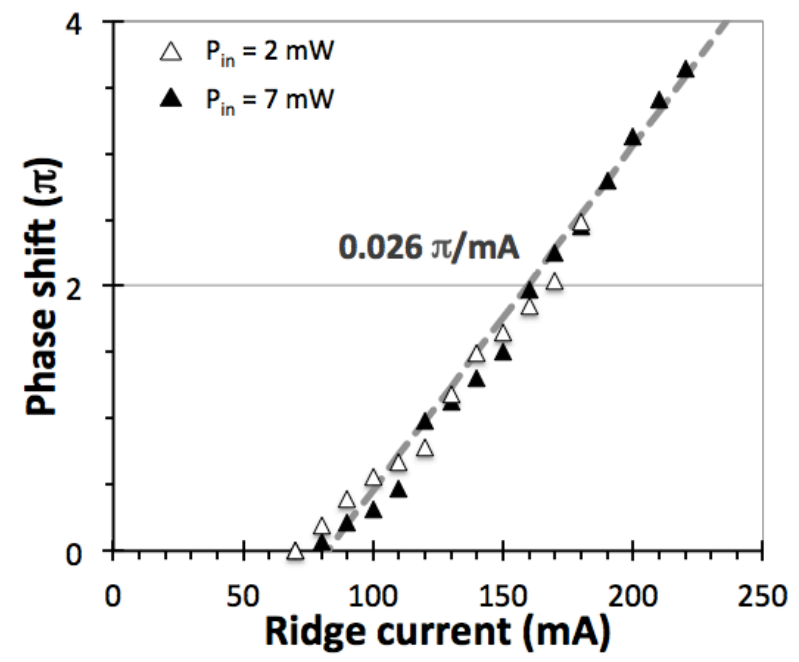

Figure 2: Shift of the fringe pattern as a function of the drive current for a 2-mm long ridge waveguide amplifier. 


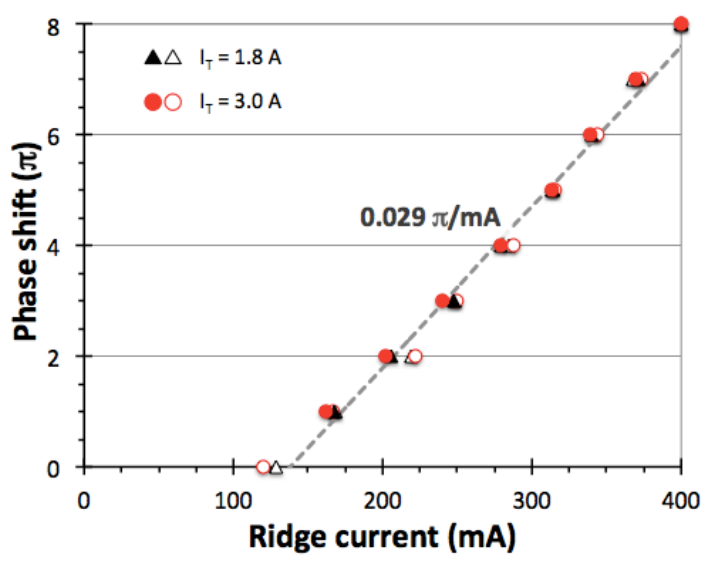

Figure 3: Shift of the fringe pattern as a function of the ridge current for a tapered amplifier, measured at the taper currents $\mathrm{I}_{\mathrm{T}}=1.8 \mathrm{~A}$ (black triangles) and $3.0 \mathrm{~A}$ (red circles) and for two different injected optical powers $\mathrm{P}_{\mathrm{in}}=1.5 \mathrm{~mW}$ (empty) and $\mathrm{P}_{\text {in }}=10 \mathrm{~mW}$ (full)

\section{PHASE-LOCKING \& COHERENT COMBINING}

\subsection{Description of the experimental set-up}

The external cavity designed for two lasers is based on a Michelson interferometer: the two laser beams are combined on a 50/50 beamsplitter (BS), and a mirror on one arm closes the external cavity (Figure 4). The two lasers share the same external cavity, and they undergo minimum losses when the two laser beams are in phase at the beamsplitter, so that interference is constructive on the $\mathrm{P}$ arm, and destructive on the other arm. On the other hand, the incoherent operation of the two lasers induces $75 \%$ losses per roudtrip in the cavity for each laser. The coherent operation of the two lasers is thus strongly favoured by the Michelson external cavity. The two lasers are collimated on both sides with high NA aspheric lenses. The rear mirror, which closes the cavity, can be either a high-reflectivity mirror, or a diffraction grating at the Littrow incidence to control the laser wavelength. On the front facet, a similar Michelson configuration is implemented with one beamsplitter. A phase plate $\mathrm{L}_{*}-$ a simple anti-reflection coated plane silica plate - is added on one beam to adjust the phase relationship between the two laser beams, as their optical paths are different. Rotating the plate allows fine tuning of the phase difference, and maximizing of the combined power in the $\mathrm{P}^{\prime}$ arm.

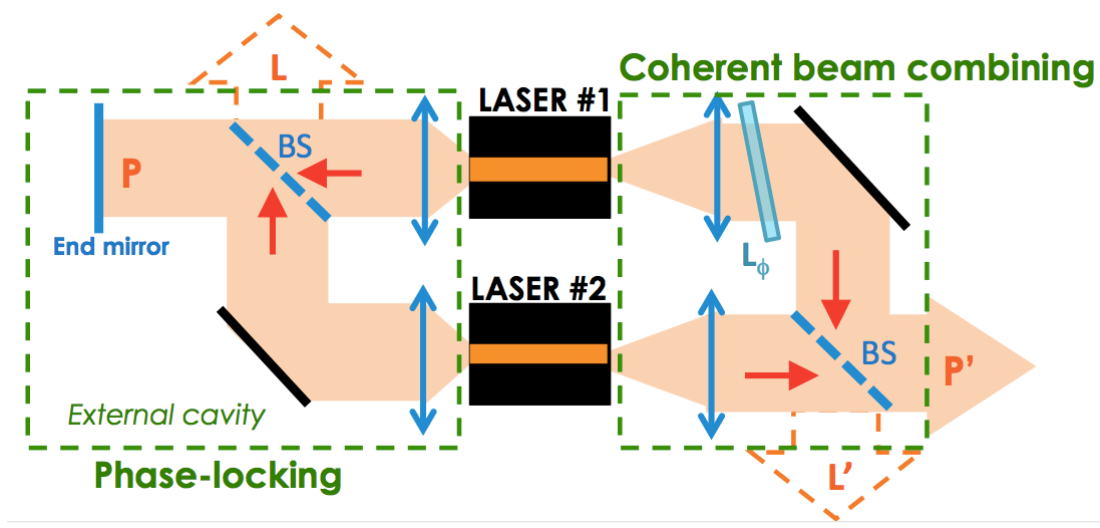

Figure 4 : Experimental set-up of the external cavity for the phase-locking - on the rear side - and the coherent combining on the front side; BS : 50/50 beamsplitter; $\mathrm{P}, \mathrm{P}^{\prime}$ : useful arms; $\mathrm{L}, \mathrm{L}^{\prime}$ : losses.

In order to clarify the following experiments, it is useful to write the standard equations describing the interference between two beams incident on a 50/50 beamsplitter with powers $P_{1}, P_{2}$, phases $\varphi_{1}, \varphi_{2}$ and relative coherence $g_{12}$ [9]. After reflection/transmission on the beamsplitter, the interference patterns on each interferometer arm are given by:

$$
P_{P}=\frac{1}{2}\left(P_{1}+P_{2}+2 \sqrt{P_{1} \times P_{2}} \times g_{12} \times \cos \left(\varphi_{2}-\varphi_{1}\right)\right)
$$




$$
P_{L}=\frac{1}{2}\left(P_{1}+P_{2}-2 \sqrt{P_{1} \times P_{2}} \times g_{12} \times \cos \left(\varphi_{2}-\varphi_{1}\right)\right)
$$

where $\mathrm{P}, \mathrm{P}^{\prime}$ are the useful arms of the interferometer in our setup and L, L' are the arms associated with losses. The ratio of the powers on the $\mathrm{L}$ and $\mathrm{P}$ arms of the external cavity provides a measurement of the phase-locking efficiency $\gamma$ :

$$
\gamma=1-\frac{\mathrm{P}_{\mathrm{L}}}{\mathrm{P}_{\mathrm{P}}}
$$

We can also define a combining efficiency $\eta_{P}$, measuring the proportion of the extracted power on the combining arm P:

$$
\eta_{P}=\frac{P_{P}}{P_{L}+P_{P}}
$$

$\eta_{P}$ and $\gamma$ are related by $\eta_{P}{ }^{-1}=2-\gamma$. Both parameters are equal to 1 if only the two laser beams are coherent and the beams are combined on the $\mathrm{P}$ arm, corresponding to $\mathrm{P}_{\mathrm{L}}=0$. Moreover, $\eta_{P}$ and $\gamma$ are equals for coherence values above typically $80 \%$ with $\mathrm{P}_{1}=\mathrm{P}_{2}$. Nevertheless, when the two lasers are incoherent and $\mathrm{P}_{1}=\mathrm{P}_{2}, \eta_{P}=50 \%$ and $\gamma=0$.

Finally, the visibility of the interferences, defined as:

$$
\mathrm{V}=\frac{\max \left(\mathrm{P}_{\mathrm{P}}\right)-\min \left(\mathrm{P}_{\mathrm{P}}\right)}{\max \left(\mathrm{P}_{\mathrm{P}}\right)+\min \left(\mathrm{P}_{\mathrm{P}}\right)}
$$

is a useful quantity and equals to the relative coherence $g_{12}$ of the two lasers when $\mathrm{P}_{1}=\mathrm{P}_{2}$.

Last but not least, we note that the interference at the BS on the rear side forces the phase relationship $\varphi_{2}=\varphi_{1}$. On the front side, however, we only have access to the phase difference $\Delta \varphi^{\prime}=\left(\varphi^{\prime}{ }_{2}-\varphi^{\prime}{ }_{1}\right)$ which differs from $\Delta \varphi$ by a constant factor that is dependent on the laser frequency $v_{\mathrm{L}}$, the geometrical distances between the lasers and the beamsplitters on both sides, and the optical path lengths through the lasers:

$$
\Delta \varphi^{\prime}=\Delta \varphi+\frac{2 \pi}{c} v_{L}\left[(n L)_{2}-(n L)_{1}\right]+\frac{2 \pi}{c} v_{L}\left(\Delta L^{\prime}-\Delta L\right)
$$

Adjusting the orientation of the phase plate on the front side permits correction of the optical path difference, so that $\Delta \varphi^{\prime}$ $=0$ can be achieved as well. Nevertheless, any change in the laser frequency $v_{\mathrm{L}}$ will modify this correction.

\subsection{Phase-locking and coherent combining of two ridge lasers}

Our experimental setup for separate phase-locking and coherent combining of two lasers was first evaluated with two ridge lasers described in $\$ 2.1$, with anti-reflection coating on the back facet and a 3\% anti-reflection coating on the front facet.

Phase-locking on the back side

The phase-locked operation of the two lasers was observed over a wide range of operating currents up to $180 \mathrm{~mA}-$ roughly three times the laser threshold. At higher currents, the external cavity is not able to force the phase-locked operation of the lasers, and they operate independently in the external cavity. This behavior is observed in the map in Figure 5 which measures the phase-locking efficiency $\gamma$ as a function of the different currents and is deduced from the measurement of $\mathrm{P}_{\mathrm{P}}$ and $\mathrm{P}_{\mathrm{L}}$ on the rear side of the laser cavity. As minimum losses are achieved when the optical powers $\mathrm{P}_{1}$ and $\mathrm{P}_{2}$ are equal on the beamsplitter, the phase-locking map exhibits lines of maximum efficiency for currents corresponding to $P_{1} \cong P_{2}$. The phase-locking efficiency $\gamma$ (as defined in equation (2)) reaches $97 \%$, and is equal in that configuration to the combining efficiency $\eta_{P}$ on the $\mathrm{P}$ arm of the interferometer. It is limited by the residual mismatch between the two beam profiles [10]. 


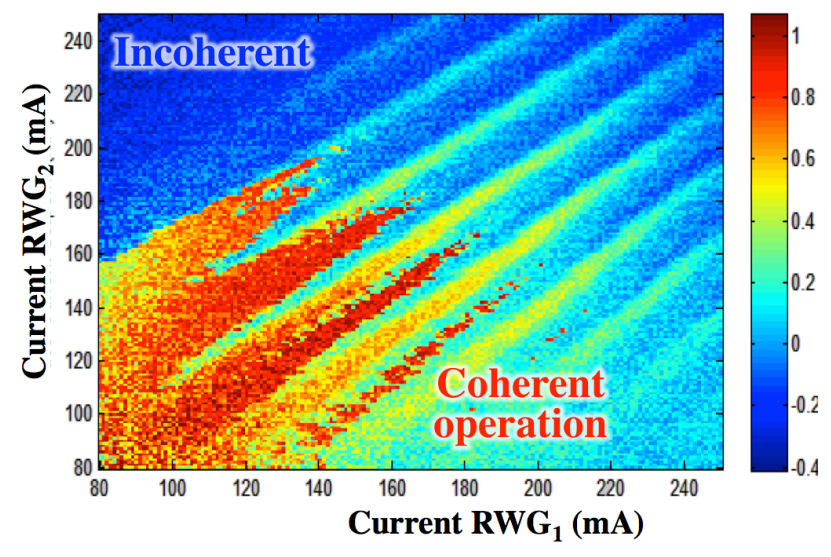

Figure 5: Map of the phase-locking efficiency $\gamma$ (on the rear facet) as a function of the operating currents of the two lasers.

Under phase-locked operation, the laser line is narrow $(<25 \mathrm{pm})$ and corresponds to the single-frequency operation of both lasers in the external cavity, as expected. However, the wavelength is not stabilized, and the laser line jumps from one mode to the other, depending on the operating conditions.

Finally, we have evaluated the robustness of the passive phase-locking to perturbations. The current of one laser was changed linearly over time, while the other one was kept constant - resulting in a known phase perturbation to the cavity. The maximum amplitude of the current change is $10 \mathrm{~mA}-$ less than $10 \%$ of the driving current. Experimentally, we observe that, depending on the operating points, the external cavity can resist current change up to $2 \mathrm{~mA}$ - corresponding to roundtrip phase perturbations of $\pi / 10$; the average tolerable phase perturbation is $\pi / 20$ at $I_{R 1} \cong I_{R 2}=140 \mathrm{~mA}$ (Figure 7).

\section{Coherent combining on the front side}

On the front side of the two lasers, adjusting the orientation of the phase plate allows the maximization the output power on the $\mathrm{P}^{\prime}$ arm, and thus, the combining efficiency $\eta^{\prime}$ as defined in Eq. (3). $\eta^{\prime}$ is above $90 \%$ over the whole range of measurement, and reaches $94 \%$ at $180 \mathrm{~mA}$-corresponding to an average relative coherence $\mathrm{g}_{12}$ of $88 \%$ between the two beams. The maximum combined power is $178 \mathrm{~mW}$, which is $85 \%$ of the maximum power extracted from both lasers operating separately in an optimized external cavity. The discrepancy between these two ratios is due to increased losses for the two lasers in the interferometric cavity which result in a higher laser threshold, as seen in Figure 6. Without any stabilization of the laser cavity and in a standard laboratory environment, we observe that the output power typically remains stable for $3 \mathrm{~min}$ before the phase relationship between the two laser beams on the front side jumps to another phase state. These phase jumps are the result of mode-hopping of the laser line from one longitudinal mode of the external cavity to another - resulting in a change in the phase shift of the laser beams through each device.

In order to measure the time evolution of the phase relationship $\Delta \varphi^{\prime}=\left(\varphi^{\prime}{ }_{1}-\varphi^{\prime}{ }_{2}\right)$ on the front side of the lasers, the two beams were slightly angled to produce interference fringes. Two photodiodes were positioned at a distance of a quarter of the interference period, giving access to fluctuations in phase (I) and quadrature phase (Q) - the inverse tangent of the ratio of I and Q unambiguously yields the phase difference $\Delta \varphi^{\prime}$. Thus, during the experiment described above, in which the external cavity operation is perturbed by changing the driving current of one laser, we were able to simultaneously monitor $\Delta \varphi^{\prime}$ (Figure 7). We observe some fast changes in the phase relationship associated to jumps in the laser line, but it appears that the phase difference $\Delta \varphi^{\prime}$ between the two lasers may recover even after the two lasers operate incoherently for a while. Consequently, for a total perturbation amplitude of $\sim \pi / 3$ corresponding to the current ramp in Figure 7, we see only two distinct phase states chosen by the external cavity. 


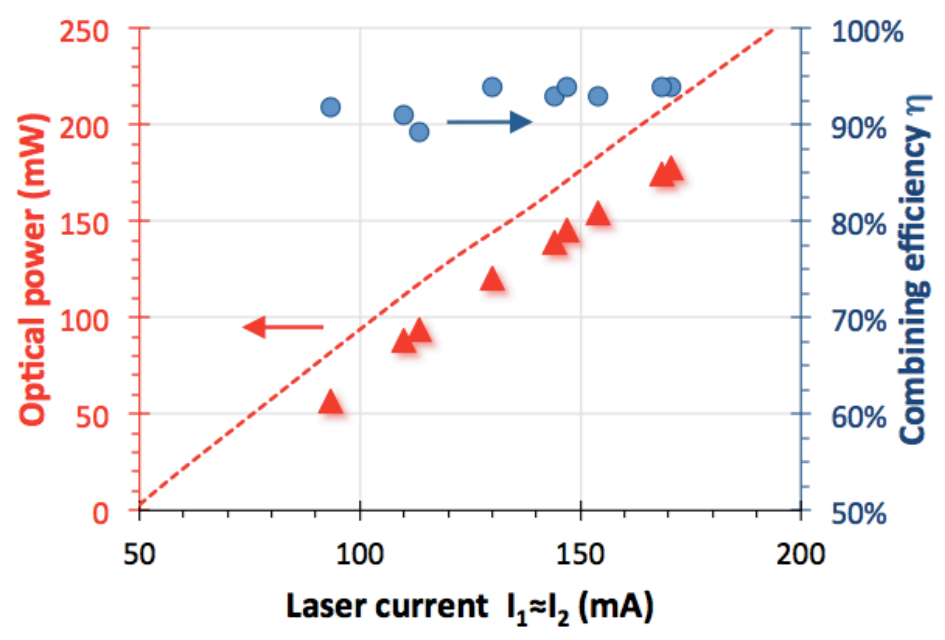

Figure 6: Coherently combined power (red triangles) and combining efficiency $\eta$ (blue circles) as a function of the operating current of the two lasers; the red line corresponds to the sum of the output powers extracted from each laser independently.

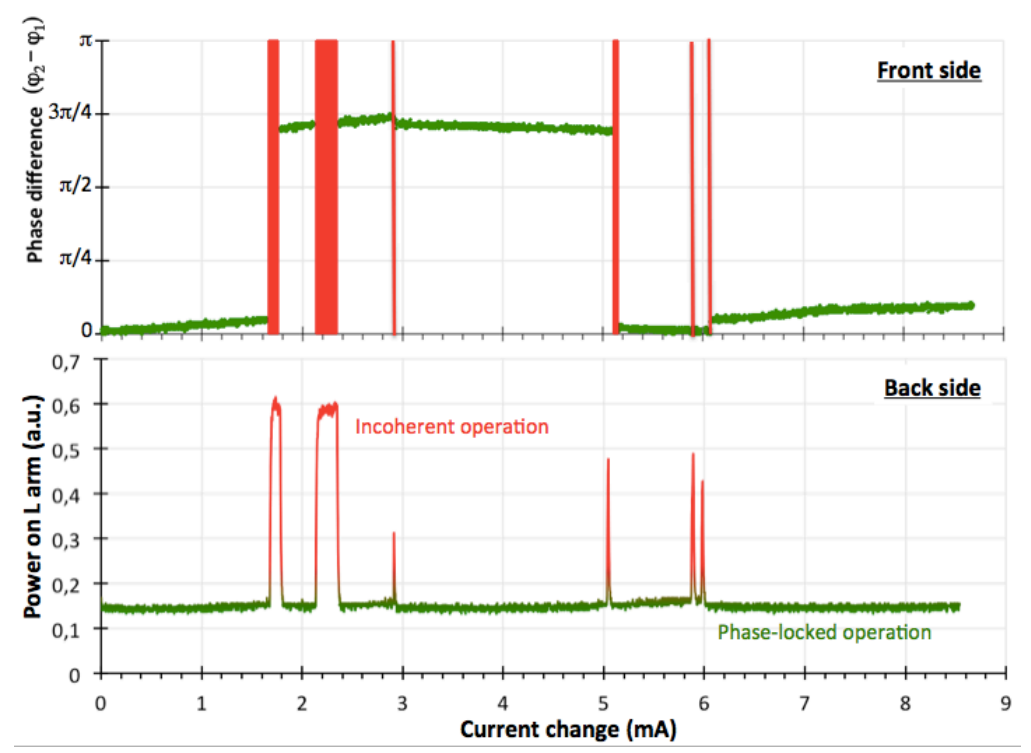

Figure 7: (bottom) Evolution of the power on the $\mathrm{L}$ arm (back side) as a function of the current change in one laser for $\mathrm{I}_{1} \cong$ $\mathrm{I}_{2} \cong 140 \mathrm{~mA}$; the two lasers are phase-locked when the losses $\mathrm{L}$ are minimum - green zone of the graph;

(top) Evolution of the phase difference on the front side, measured during the same current change.

\section{Passive and active stabilization of the coherent combining efficiency}

To maximize the combined power on the $\mathrm{P}^{\prime}$ arm, the orientation of the phase plate $\mathrm{L}_{\phi}$ has to be corrected after each phase shifts between the lasers on their front facet. With the aim to automatically maintain the coherent combining efficiency $\eta^{\prime}$ to its highest value, we have compared two techniques: one relies on a passive optical feedback and the other one on an active correction of the driving currents.

The passive optical feedback is implemented with a low-reflection (1\%) plane plate positioned in the common output beam P'. It provides a weak feedback into the lasers which favors a phase relationship $\Delta \varphi^{\prime}=\left(\varphi^{\prime}{ }_{1}-\varphi^{\prime}{ }_{2}\right)$ resulting in constructive interferences on the front beamsplitter. It is noteworthy that this low optical feedback is not sufficient to phase-lock the two emitters by itself. Experimentally, this passive feedback ensures the long-term ( $>15 \mathrm{mn})$ stabilization of the combined power, minimum losses on the extracted power and RMS fluctuations of the combined power below 
$0.5 \%$. The external cavity self-adjusts when the laser currents are modified and the combining efficiency $\eta^{\prime}$ remains between $75 \%$ and $85 \%$ depending on the driving currents (Figure 8 ).

Secondly, automatic correction of the laser currents has been used. The power L' on the front side is measured at a $10 \mathrm{~Hz}$ rate, and the currents are automatically changed to minimize it. The minimization algorithm uses a random exploration of the currents around its nominal value, with a $0.5 \mathrm{~mA}$ maximum amplitude. Due to the highly non-linear behavior of the laser cavity, it is noteworthy that the currents are not optimized, strictly speaking - as opposed to the SPGD protocol used in MOPA configurations [3]. Instead, they are corrected in order to ensure the proper phase relationship on the front side, while the external cavity self-adjusts to maintain the phase-locking. The active correction only runs if the L' power increases above a fixed threshold, otherwise it is switched off. This long-term electronic correction of the operating currents complete the passive optical feedback by the external cavity on the rear side and stabilize the coherent beam combining efficiency $\eta^{\prime}$, which remains above $93 \%$.

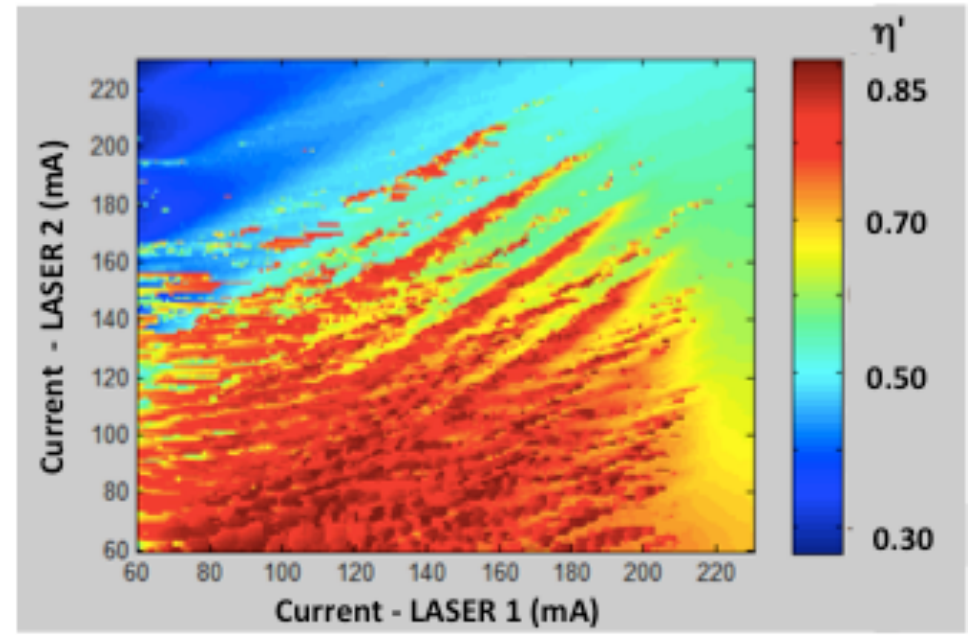

Figure 8: Map of the combining efficiency $\eta$ ' on the front facet as a function of the operating currents of the two lasers, with a weak optical feedback.

\subsection{Phase-locking and coherent combining of two tapered lasers}

The experimental setup as in Figure 4 was also implemented with two high-brightness tapered lasers. For such lasers, the separate phase-locking - on the rear side - and coherent combining on the front side seems particularly adequate, as optical feedback on the front side may deteriorate their beam quality or even destroy them. On the ridge (rear) side, the laser beams are collimated with high NA aspheric lenses, while on the taper (front) side, an aspheric lens and a cylindrical lens are used to correct their intrinsic astigmatism. A 2000 lines/mm grating is used at the Littrow incidence to close the external cavity on the back side. This ensures a stable and narrow laser line at $966.9 \mathrm{~nm}$. The common external cavity on the rear side of the two tapered devices ensures their passive phase-locking for currents up to $\mathrm{I}_{\mathrm{T}}=6 \mathrm{~A}$. The phase-locking efficiency $\eta$ reaches $60 \%$ and was limited at the time of the experiment by the improper alignment of the lenses. On the front side, the laser power extracted from each device is $3 \mathrm{~W}$ at $\mathrm{I}_{\mathrm{R}}=200 \mathrm{~mA}$ and $\mathrm{I}_{\mathrm{T}}=6 \mathrm{~A}$. Under these conditions, the maximum combined power at the $\mathrm{P}^{\prime}$ output of the front interferometer is $4.2 \mathrm{~W}-$ corresponding to $\eta_{P}^{\prime}=$ $70 \%$ of the total output power. When the two laser beams are superposed with a slight angle, we observe high-contrast interference fringes, with a visibility $\mathrm{V} \geq 90 \%$ in the center (Figure 9). Such a high visibility demonstrates that the coherence between the two lasers beams is extremely good. Thus, the poor combining efficiency achieved is only limited by the wavefront and intensity mismatches between the two laser beam profiles [10]. We are currently investigating these limitations. These results are promising, as they demonstrate the efficient coherent combining of two highbrightness high-power laser diodes in a simple architecture. 


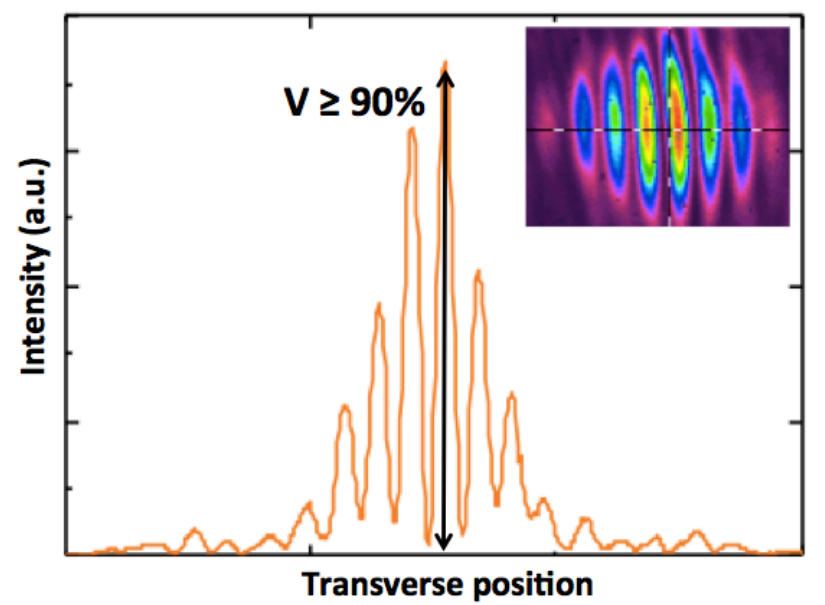

Figure 9: Transverse profile of the interferences between the two laser beams from the front side of the tapered laser diodes in the slow-axis direction; the output power from each laser is $3 \mathrm{~W}$.

\section{CONCLUSION}

We have proposed a new coherent beam combining architecture designed for high-brightness diode lasers, in which the lasers are passively phase-locked by use of an external cavity on their back facet, while the coherent combining is realized on the front side with limited extraction losses. This arrangement leads to a coherent beam combining efficiency comparable to that achieved in a MOPA configuration.

Our setup was implemented first with two ridge lasers, in a Michelson-type cavity. We observe that, although the two lasers are phase-locked by the external cavity, their phase relationship may jump because of a longitudinal mode hop, resulting in an apparent reduction of the coherent combining efficiency. In order to stabilize the relative laser phases on their front side, we have compared two feedback configurations - one using a weak optical feedback from the combined output, the other based on the automatic exploration of the driving currents around their nominal values. Both have resulted in the long-term stabilization of the combined power, with an efficiency up to $93 \%$.

Finally, we evaluated our architecture with two tapered laser diodes. More than $4 \mathrm{~W}$ of combined power has been achieved from two devices with an efficiency of $70 \%$, showing its capability to extend phase-locked operation towards high power levels. We have demonstrated that the combining efficiency was most certainly limited by wavefront and intensity mismatches between the two combined laser beams, as their relative coherence is greater than $90 \%$.

\section{REFERENCES}

[1] Fan, T.Y, "Laser beam combining for high-power, high-radiance sources", IEEE Journal of Selected Topics in Quantum Electronics 11 (3), 567-577 (2005)

[2] Brignon, A., [Coherent Laser beam combining], Wiley - VCH (2013)

[3] Redmond, S. M., Creedon, K. J., Kansky, J. E., Augst, S. J., Missaggia, L. J., Connors, M. K., Huang, R. K., Chann, B. , Fan, T. Y., Turner, G. W. and Sanchez-Rubio, A., "Active coherent beam combining of diode lasers", Optics Letters 36 (6), 999-1001 (2011)

[4] Creedon, K. J., Redmond, S. M, Smith, G.M., Missaggia, L. J., Connors, M. K., Kansky, J. E., Fan, T. Y., Turner, G. W. and Sanchez-Rubio, A., "High efficiency coherent beam combining of semiconductor optical amplifiers", Optics Letters 37 (23), 5006-5008 (2012)

[5] Corcoran, C. J. and Durville, F., "Passive coherent combination of a diode laser array with 35 elements", Optics Express 22 (7), $8420-8425$ (2014)

[6] Montoya, J., Augst, S. J., Creedon, K. J., Kansky, J. E., Fan, T. Y. and Sanchez-Rubio, A., "External cavity beam combining of 21 semiconductor lasers using SPGD", Applied Optics 51(11), 1724-1728 (2012) 
[7] Fiebig, C., Blume, G., Kaspari, C., Feise, D., Fricke, J., Matalla, M., John, W., Wenzel, H., Paschke, K. and Erbert G., "12W high-brightness single-frequency DBR tapered diode laser" Electron. Lett 44 (21), 1253-1255 (2008).

[8] Larkins, E.C., Bull, S., Kaunga-Nyirenda, S.N., Helal, M.A., Vilokkinen, V., Uusimaa, P., Crump, P. and Erbert, G., "Design Optimisation of High-Brightness Laser Diodes for External Cavity Operation in the BRIDLE Project," 24 ${ }^{\text {th }}$ IEEE Semiconductor Laser Conference (Palma de Mallorca, 7 September 2014).

[9] Saleh, B. E. A., Teich, M. C. [Fundamentals of Photonics], Wiley Series in Pure and Applied Optics (2013)

[10] Goodno, G. D., Shih, C.-C., Rothenberg, J. E., "Perturbative analysis of coherent combining efficiency with mismatched lasers " Optics Express 18 (24), 25403-254014 (2010)

\section{Acknowledgments}

This work was supported in part by the European Commission within the BRIDLE program $\left(7^{\text {th }}\right.$ FP) under grant 314719. We thank Gunnar Blume from the FBH for detailed technical discussions and for providing the tapered laser devices used in this investigation. 